\title{
Consumo alimentar e sua associação com estado nutricional, atividade física e fatores sociodemográficos de candidatos à cirurgia bariátrica.
}

\section{Food consumption and its association with nutritional status, physical activity and sociodemographic factors of bariatric surgery candidates.}

\author{
Renata Adrielle lima Vieira ${ }^{1}$; $;$ Lucio Vilar Rabelo Filho²; Maria Goretti Pessoa de Araújo Burgos³
}

R E S U M O

\begin{abstract}
Objetivo: avaliar a associação do consumo alimentar com estado nutricional, atividade física e fatores sociodemográficos no pré-operatório de cirurgia bariátrica. Métodos: estudo transversal, realizado no período de 2018 a 2019, com pacientes internados para a cirurgia bariátrica no Hospital Universitário Oswaldo Cruz da Universidade Federal de Pernambuco. Foram obtidos dados sociodemográficos, estado nutricional pelo índice de massa corporal (IMC), circunferência da cintura, maior peso e peso pré-cirúrgico, consumo alimentar obtido pelo questionário de frequência alimentar baseado na pirâmide alimentar adaptada para o paciente bariátrico e atividade física. Resultados: participaram do estudo 60 pacientes, sendo $78,3 \%$ do sexo feminino, com idades de 38,8 89,6 anos, $53,3 \%$ casados e $70 \%$ dos indivíduos com mais de 12 anos de estudo. Na prática de atividade física, 31,7\% eram sedentários. Sobre o estado nutricional, a média de IMC observada foi de $47,3 \pm 6,96 \mathrm{~kg} / \mathrm{m}^{2}$. O peso pré-operatório, maior peso atingido antes da cirurgia e circunferência da cintura foi maior no sexo masculino $(p<0,05)$. Houve associação entre atividade física e consumo alimentar, sendo que indivíduos sedentários consumiam mais carboidratos $(p=0,041)$. Por outro lado, dados sociodemográficos e estado nutricional não apresentaram associações com o consumo. Conclusão: o nível de atividade física evidenciou associação significativa apenas com o consumo de carboidratos.
\end{abstract}

Descritores: Cirurgia Bariátrica. Obesidade. Consumo de Alimentos. Estado Nutricional. Período Pré-Operatório.

\section{INTRODUÇÃO}

A obesidade é uma doença complexa e multifatorial, em crescimento exponencial no Brasil e no mundo. É considerada fator de risco para doenças cardiovasculares, diabetes mellitus tipo 2, hipertensão, dislipidemia e múltiplos cânceres, sendo causada pela interação de fatores genéticos, ambientais, metabólicos, psicológicos e comportamentais ${ }^{1}$. Seu tratamento é complexo e envolve mudança de estilo de vida e tratamento farmacológico. Entretanto, estes têm sido pouco efetivos em obesos mórbidos e não se mostram sustentáveis em longo prazo². É consenso que a cirurgia bariátrica é o método mais efetivo para o tratamento e profilaxia das complicações causadas pela obesidade mórbida, sendo indicado quando o tratamento conservador é ineficaz ${ }^{3-5}$.
Dentre os efeitos da cirurgia, se destaca a redução do peso, com melhora do quadro metabólico, que resulta na diminuição do risco cardiovascular, da resistência à insulina, do diabetes, entre outros ${ }^{6}$. No entanto, carências podem ocorrer e estão relacionadas à ingestão alimentar reduzida, baixa adesão aos suplementos, má absorção de nutrientes no pós-operatório, e também às deficiências existentes antes da cirurgia ${ }^{7,8}$. Esse quadro de deficiência pode acarretar anemia, osteoporose, distúrbios neurológicos e desnutrição .

Sobre as características do consumo alimentar de macronutrientes de pacientes obesos, observa-se elevada ingesta calórica oriunda de carboidratos, gorduras trans e saturadas, associada à diminuição do consumo de frutas, verduras e proteínas, o que resulta no ganho de peso corporal ${ }^{9}$.

1 - Universidade Federal do Pernambuco, Centro de Ciências Médicas, Faculdade de Medicina, Departamento de Medicina Clínica, Programa de Pós-Graduação em Cirurgia, Recife, PE, Brasil. 2 - Universidade Federal do Pernambuco, Centro de Ciências Médicas, Faculdade de Medicina, Departamento de Medicina Clínica, Recife, PE, Brasil. 3 - Universidade Federal do Pernambuco, Centro de Ciências Médicas, Faculdade de Medicina, Departamento de Nutrição, Recife, PE, Brasil. 
Com o intuito de intervir em tempo hábil e evitar complicações clínicas e nutricionais mais graves após a cirurgia, se faz necessário promover práticas alimentares e de hábitos de vida saudáveis ${ }^{10}$. Portanto, o objetivo deste estudo foi verificar a associação do consumo alimentar com estado nutricional, atividade física e fatores sociodemográficos de candidatos à cirurgia bariátrica.

\section{MÉTODOS}

Estudo transversal realizado com pacientes no pré-operatório de cirurgia bariátrica do Hospital Universitário Oswaldo Cruz da Universidade Federal de Pernambuco (HUOC-UPE), Recife/PE, no período de 2018 a 2019. Utilizou-se como critérios de elegibilidade pacientes de ambos os sexos, com idades entre 18 e 59 anos. Aqueles que apresentaram histórico de doença psíquica, uso de drogas ilícitas, risco cirúrgico elevado, deficientes físicos por impossibilidade de realizar antropometria, que apresentavam intervenção cirúrgica pregressa do trato digestório, internados para reabordagem cirúrgica por reganho de peso e aqueles que não sabiam ler e escrever, foram excluídos do estudo.

Dados sociodemográficos, como idade, sexo, escolaridade em anos de estudo ${ }^{11}$, estado civil e procedência, foram obtidos, assim como, medidas antropométricas de peso pré-operatório $(\mathrm{Kg})$, maior peso atingido $(\mathrm{Kg})$, altura $(\mathrm{m})$ e circunferência da cintura (CC) pré-operatória $(\mathrm{cm})^{12}$. A CC foi aferida utilizando-se uma fita métrica flexível e inelástica, dividida em centímetros e subdivida em milímetros (com precisão de $1 \mathrm{~mm}$ ). Para a aferição, a fita métrica foi localizada a cerca de $2 \mathrm{~cm}$ acima da cicatriz umbilical devido à dificuldade de obter o ponto médio entre a última costela e a crista ilíaca nesses pacientes ${ }^{13}$. Após obtenção do peso e altura, foi calculado o índice de massa corporal (IMC), sendo utilizado os pontos de corte recomendados pela American Society for Metabolic \& Bariatric Surgery².
Para avaliar o nível de atividade física, utilizou-se o Questionário Internacional de Atividade Física - IPAQ, em sua versão curta, que classifica os indivíduos em muito ativos, ativos, irregularmente ativos e sedentários ${ }^{14}$. O consumo alimentar foi estimado pelo questionário de frequência alimentar direcionado (QFAD) e adaptado, desenvolvido por Soares et al. ${ }^{15}$, baseado nos parâmetros da pirâmide alimentar para pacientes bariátricos proposto por Moizé et al. ${ }^{16}$. Esta pirâmide divide-se em cinco níveis, sendo sua base relacionada à ingestão hídrica e de suplementos, além da prática de atividade física. Os outros quatro níveis abordam sobre o consumo de grupos alimentares, sendo grupo 1: proteínas (leguminosas, carnes, ovos, leite e derivados); grupo 2: hortaliças, frutas e azeite de oliva; grupo 3: arroz, massas, raízes e tubérculos; grupo 4: bebidas alcoólicas, industrializados, alimentos ricos em açúcar, gorduras saturadas, trans e colesterol.

A avaliação pelo QFAD divide os alimentos distribuídos nos quatro grupos alimentares presentes nesta pirâmide, segundo frequências categorizadas de consumo em: raro ou nunca, uma a três vezes no mês, uma vez na semana, duas a quatro vezes na semana, cinco ou mais vezes na semana, uma vez ao dia, duas ou mais vezes por dia. O questionário aborda dados referentes ao mês anterior à entrevista a fim de evitar relatos superestimados e auxiliar na recordação do consumo.

A avaliação do consumo foi realizada com base na metodologia proposta por Fornés et al. ${ }^{17}$, na qual o cálculo geral da frequência de consumo é convertido em escores. Para transformar as frequências relatadas no QFAD de cada alimento em frequência diária, foi atribuído um peso a cada categoria de frequência de consumo, considerando como referência o consumo relatado "uma vez por dia" igual a 1. Dessa forma, para frequência "raro ou nunca" o correspondente em frequência diária foi 0; para " 1 a 3 vezes no mês" foi atribuído 0,067 (2x/30 dias); 
para " 1 vez na semana" foi 0,143 (1x/7 dias); para "2 a 4 vezes na semana" 0,429 (3x/7 dias); para

" 5 a 6 vezes na semana" 0,786 (5,5x/7 dias); para "2 ou mais vezes por dia" foi igual a 2. Além disso, foi avaliado também a utilização de suplementos polivitamínicos e minerais prévios à cirurgia bariátrica e ingestão hídrica.

Para as análises estatísticas foi empregado o pacote estatístico SPSS versão 12.0 (SPSS Inc., Chicago, IL, USA). Inicialmente, as variáveis contínuas foram testadas segundo a normalidade pelo teste de Kolmogorov-Smirnov. As variáveis com distribuição normal foram descritas pela média e desvio padrão e aquelas com distribuição não paramétricas, foram descritas pela mediana e o respectivo intervalo interquartílico (IQ) (Percentis 25 e 75). A comparação entre as médias foi obtida pelo teste $t$ de Student (duas médias). Os escores de frequência de consumo alimentar, por se tratarem de variáveis em escala ordinal, foram descritos na forma de mediana e IQ. A associação entre o consumo alimentar e as variáveis independentes foi avaliada pelos testes u de Mann-
Whitney (duas medianas) e Kruskal-Wallis (mais de duas medianas) e empregou-se o teste u de MannWhitney a posteriori. Na validação das associações investigadas foi adotado o valor de $p<0,05$.

Todos os participantes foram informados sobre o trabalho, receberam por escrito uma descrição do estudo e de todos os procedimentos a que foram submetidos e assinaram o Termo de Consentimento Livre e Esclarecido (TCLE). Esta pesquisa foi aprovada pelo Comitê de Ética em Pesquisa em Seres Humanos do Centro de Ciências da Saúde (CCS) da Universidade Federal de Pernambuco, de acordo com a Resolução $n$ 으 466/12, do Conselho Nacional de Saúde, sob o parecer CAAE: 67051817.9.0000.5192.

\section{RESULTADOS}

Foram avaliados 60 pacientes submetidos à cirurgia bariátrica, com uma média de idade de $38,8 \pm 9,6$ anos, sendo $78,3 \%$ do sexo feminino. Dados sobre o perfil sociodemográfico dos pacientes estão apresentados na tabela 1.

Tabela 1. Perfil sociodemográfico dos pacientes antes da cirurgia bariátrica (HUOC-UPE).

\begin{tabular}{lcc}
\hline \multicolumn{1}{c}{ Característica } & $\mathrm{n}$ & $\%$ \\
\hline Sexo & & 21,7 \\
Masculino & 13 & 78,3 \\
$\quad$ Feminino & 47 & \\
Estado civil & & 53,3 \\
$\quad$ Casado(a) & 32 & 22,0 \\
Solteiro(a) & 23 & 6,0 \\
Separado(a) ou divorciado(a) & 5 & 38,3 \\
Cidade & & 58,3 \\
Recife/RMR* & 23 & 3,4 \\
Interior do Estado & 35 & \\
Outros Estados & 2 & 6,7 \\
Escolaridade & & 11,7 \\
$<4$ anos de estudo & 4 & 11,7 \\
$4-8$ anos de estudo & 7 & 70,0 \\
$9-11$ anos de estudo & 7 & \\
$\geq 2$ anos de estudo & 42 & \\
\hline
\end{tabular}

* $R M R=$ região metropolitana do Recife. 
Com relação ao estado nutricional, foi encontrada diferença estatisticamente significante entre média do peso pré-operatório, maior peso atingido antes da cirurgia e circunferência da cintura entre os sexos, sendo superior no sexo masculino $(p<0,05)$. A média de IMC observada foi de $47,3 \pm 6,96 \mathrm{~kg} / \mathrm{m}^{2}$ (Tabela 2).

Quanto à atividade física, foi observado que 24 pacientes (40\%) eram ativos, 17 $(28,3 \%)$ insuficientemente ativos, e 19 (31,7\%) sedentários. Além disso, no que se refere ao uso de suplementação de vitaminas e/ou minerais antes da cirurgia, 20 (33,3\%) usaram em algum momento, especialmente polivitamínicos e minerais, citrato de cálcio e vitamina D. Quanto à ingestão hídrica, a maioria ingeria mais de dois litros de água/dia $(71,7 \%)$ (dados não apresentados em tabelas). Não foram encontradas diferenças significativas entre os escores de consumo alimentar dos pacientes candidatos à cirurgia bariátrica com dados sociodemográficos (Tabela 3). Porém, observouse que indivíduos sedentários apresentaram maior consumo de alimentos pertencentes ao grupo alimentar dos carboidratos $(p=0,041)$ (Tabela 4).

Tabela 2. Variáveis antropométricas dos pacientes antes da cirurgia bariátrica (HUOC-UPE).

\begin{tabular}{lcccc}
\hline \multirow{2}{*}{ Variáveis } & Sexo feminino $\mathrm{n}=47$ & Sexo masculino $\mathrm{n}=13$ & Total $\mathrm{n}=60$ & $p$-valor \\
\cline { 2 - 5 } & Média $\pm \mathrm{DP}^{*}$ & Média $\pm \mathrm{DP}$ & Média $\pm \mathrm{DP}$ & \\
\hline Peso antes da cirurgia $(\mathrm{Kg})$ & $114,98 \pm 15,36$ & $148,40 \pm 26,03$ & $122,22 \pm 22,69$ & 0,001 \\
Maior peso $(\mathrm{Kg})$ & $125,48 \pm 17,16$ & $159,76 \pm 21,72$ & $132,91 \pm 22,98$ & 0,000 \\
$\mathrm{IMC} * *\left(\mathrm{~kg} / \mathrm{m}^{2}\right)$ & $46,64 \pm 6,57$ & $49,79 \pm 8,04$ & $47,3 \pm 6,96$ & 0,150 \\
$\mathrm{CC} * * *(\mathrm{~cm})$ & $122,11 \pm 12,69$ & $135,31 \pm 14,30$ & $124,97 \pm 14,04$ & 0,002 \\
\hline
\end{tabular}

* DP: desvio padrão; ** IMC: índice de massa corporal; *** CC: circunferência da cintura.

Tabela 3. Medianas e intervalos interquartílicos dos escores de consumo alimentar segundo variáveis sociodemográficas dos pacientes antes da cirurgia bariátrica (HUOC-UPE).

\begin{tabular}{|c|c|c|c|c|}
\hline \multirow{3}{*}{ Variáveis } & \multicolumn{4}{|c|}{ Grupos de alimentos } \\
\hline & Grupo 1 & Grupo 2 & Grupo 3 & Grupo 4 \\
\hline & Mediana $\left(\mathrm{IQ}^{*}\right)$ & Mediana $\left(\mathrm{IQ}^{*}\right)$ & Mediana $(\mathrm{IQ} *)$ & Mediana $(\mathrm{IQ} *)$ \\
\hline \multicolumn{5}{|l|}{ Sexo } \\
\hline Masculino & $0,64(0,28-0,64)$ & $0,69(0,35-1,00)$ & $0,89(0,35-1,01)$ & $0,68(0,27-1,16)$ \\
\hline Feminino & $0,57(0,44-0,80)$ & $0,75(0,50-1,00)$ & $0,76(0,48-1,00)$ & $0,37(0,09-0,80)$ \\
\hline$p$-valor & 0,872 & 0,547 & 0,971 & 0,114 \\
\hline \multicolumn{5}{|l|}{ Estado civil } \\
\hline Casado(a) & $0,53(0,42-0,88)$ & $0,73(0,47-1,00)$ & $0,76(0,45-1,00)$ & $0,41(0,21-0,80)$ \\
\hline Solteiro(a) & $0,63(0,48-0,83)$ & $0,74(0,57-0,97)$ & $0,85(0,41-1,00)$ & $0,40(0,09-0,87)$ \\
\hline Separado(a)/divorciado(a) & $0,51(0,33-0,60)$ & $0,71(0,29-1,02)$ & $0,89(0,49-1,25)$ & $0,24(0,31-1,04)$ \\
\hline$p$-valor** & 0,293 & 0,915 & 0,633 & 0,854 \\
\hline \multicolumn{5}{|l|}{ Cidade } \\
\hline Recife/RMR $* * *$ & $0,63(0,42-0,80)$ & $0,80(0,42-1,10)$ & $0,78(0,44-1,00)$ & $0,42(0,22-0,95)$ \\
\hline Interior do Estado & $0,57(0,44-0,83)$ & $0,71(0,46-1,00)$ & $0,76(0,51-1,00)$ & $0,40(0,08-0,80)$ \\
\hline$p$-valor & 0,793 & 0,372 & 0,911 & 0,171 \\
\hline \multicolumn{5}{|l|}{ Escolaridade } \\
\hline$<4$ anos de estudo & $0,46(0,35-0,54)$ & $0,55(0,28-0,91)$ & $0,57(0,34-0,81)$ & $0,49(0,17-0,78)$ \\
\hline 4-8 anos de estudo & $0,70(0,62-0,98)$ & $0,85(0,71-1,00)$ & $0,75(0,53-1,25)$ & $0,40(0,09-1,00)$ \\
\hline 9-11 anos de estudo & $0,90(0,56-1,20)$ & $1,00(0,50-1,00)$ & $1,00(0,44-1,00)$ & $0,68(0,60-1,15)$ \\
\hline$\geq 12$ anos de estudo & $0,54(0,42-0,72)$ & $0,71(0,45-1,00)$ & $0,77(0,37-1,00)$ & $0,38(0,09-0,86)$ \\
\hline$p$-valor** & 0,096 & 0,527 & 0,540 & 0,355 \\
\hline
\end{tabular}

Grupo 1= proteínas; Grupo 2= hortaliças, frutas e azeite; Grupo 3= arroz, massas, raizes e tubérculos; Grupo 4= bebida alcoólica, industrializados, alimentos ricos em açúcar, gorduras saturadas, trans e colesterol; * IQ: intervalo interquartílico. Teste u de Mann-Whitney; ** teste de Kruskal-Wallis. Teste a posteriori: u de Mann-Whitney; *** RMR: região metropolitana do Recife. 
Tabela 4. Medianas e intervalos interquartílicos dos escores de consumo alimentar segundo variáveis antropométricas e atividade física dos pacientes antes da cirurgia bariátrica (HUOC-UPE).

\begin{tabular}{|c|c|c|c|c|}
\hline \multirow{3}{*}{ Variáveis } & \multicolumn{4}{|c|}{ Grupo de alimentos } \\
\hline & Grupo 1 & Grupo 2 & Grupo 3 & Grupo 4 \\
\hline & Mediana $\left(I Q^{*}\right)$ & Mediana $\left(\mathrm{IQ}^{*}\right)$ & Mediana $\left(I Q^{*}\right)$ & Mediana $\left(\mathrm{IQ}^{*}\right)$ \\
\hline \multicolumn{5}{|l|}{ Peso antes da cirurgia } \\
\hline$<120 \mathrm{~kg}$ & $0,61(0,44-0,80)$ & $0,71(0,42-1,00)$ & $0,80(0,51-1,05)$ & $0,33(0,09-0,79)$ \\
\hline$\geq 120 \mathrm{~kg}$ & $0,57(0,41-0,86)$ & $0,75(0,53-1,00)$ & $0,76(0,41-1,00)$ & $0,38(0,17-0,75)$ \\
\hline$p$-valor & 0,819 & 0,610 & 0,051 & 0,700 \\
\hline \multicolumn{5}{|c|}{ Maior peso antes da cirurgia } \\
\hline$<130 \mathrm{~kg}$ & $0,58(0,45-0,78)$ & $0,71(0,38-1,00)$ & $0,85(0,58-1,25)$ & $0,33(0,13-0,75)$ \\
\hline$\geq 130 \mathrm{~kg}$ & $0,57(0,42-0,83)$ & $0,75(0,57-1,00)$ & $0,75(0,32-1,00)$ & $0,35(0,13-0,83)$ \\
\hline$p$-valor & 0,790 & 0,705 & 0,382 & 0,564 \\
\hline \multicolumn{5}{|l|}{$C C^{* *}$} \\
\hline$<120 \mathrm{~cm}$ & $0,54(0,44-0,72)$ & $0,75(0,33-1,00)$ & $0,75(0,42-1,00)$ & $0,35(0,18-0,69)$ \\
\hline$\geq 120 \mathrm{~cm}$ & $0,62(0,43-0,90)$ & $0,73(0,53-1,00)$ & $0,82(0,46-1,00)$ & $0,33(0,11-0,83)$ \\
\hline$p$-valor & 0,346 & 0,737 & 0,664 & 0,802 \\
\hline \multicolumn{5}{|l|}{$\mathrm{IMC} * \star \star$} \\
\hline $35-39,9 \mathrm{~kg} / \mathrm{m}^{2}$ & $0,62(0,52-0,95)$ & $0,75(0,43-1,37)$ & $0,85(0,75-1,12)$ & $0,33(0,08-0,67)$ \\
\hline $40-49,9 \mathrm{~kg} / \mathrm{m}^{2}$ & $0,58(0,46-0,90)$ & $0,74(0,46-1,02)$ & $0,77(0,41-1,03)$ & $0,44(0,12-0,85)$ \\
\hline $50-59,9 \mathrm{~kg} / \mathrm{m}^{2}$ & $0,49(0,34-0,66)$ & $0,71(0,41-1,00)$ & $0,71(0,41-1,00)$ & $0,29(0,11-0,64)$ \\
\hline$p$-valor* & 0,107 & 0,779 & 0,369 & 0,455 \\
\hline \multicolumn{5}{|l|}{ Atividade física } \\
\hline Sedentário & $0,57(0,32-1,10)$ & $0,75(0,25-1,00)$ & $0,89(0,75-1,25) a$ & $0,54(0,20-1,15)$ \\
\hline Insuficiente ativo & $0,54(0,44-0,70)$ & $0,69(0,48-1,05)$ & $0,71(0,44-0,98) b$ & $0,34(0,10-0,42)$ \\
\hline Ativo & $0,56(0,45-0,83)$ & $0,80(0,59-0,98)$ & $0,75(0,31-1,00) b$ & $0,45(0,08-0,82)$ \\
\hline$p$-valor ${ }^{\#}$ & 0,912 & 0,924 & 0,041 & 0,205 \\
\hline
\end{tabular}

Grupo 1= proteínas; Grupo 2= hortaliças, frutas e azeite; Grupo 3= arroz, massas, raízes e tubérculos; Grupo 4= bebida alcoólica, industrializados, alimentos ricos em açúcar, gorduras saturadas, trans e colesterol; * IQ: intervalo interquartílico; u de Mann-Whitney; ${ }^{*}$ CC: circunferência da cintura; *** IMC: índice de massa corporal; a,b letras diferentes, diferenças estatísticas entre as categorias; " teste de Kruskal-Wallis. Teste a posteriori: u de Mann-Whitney.

\section{DISCUSSÃO}

A velocidade de crescimento da obesidade mórbida consegue ser mais expressiva do que obesidade em geral, e isso reflete no aumento do número de cirurgias bariátricas realizadas no Brasil $^{18}$. No entanto, o paciente obeso necessita de acompanhamento em todas as etapas do processo, antes e depois da cirurgia, a fim de identificar e corrigir o consumo alimentar e/ou necessidade de suplementação, para minimizar as complicações nutricionais comuns nesse público. As complicações nutricionais mais prevalentes e relatadas na literatura são alopécia, astenia, alteração na textura das unhas, todas consideradas preditivos de carências nutricionais ${ }^{19,20}$.
O acompanhamento clínico-nutricional antes da cirurgia permite a correção destas carências e pode reduzir complicações nutricionais maiores. No presente estudo, foi observado que 33,3\% dos pacientes usaram suplementos no pré-operatório, como polivitamínicos e minerais, citrato de cálcio e vitamina D. Pacientes submetidos a procedimentos cirúrgicos disabsortivos devem utilizar estes suplementos de forma preventiva, pois, além das alterações anatômicas e fisiológicas que prejudicam a absorção e/ou ingestão alimentar, seu uso no pós-operatório costuma ser escasso e esporádico, implicando em queda no estado nutricional do paciente ${ }^{8,21}$.

A maioria dos pacientes avaliados era do sexo feminino. Isso corrobora com vários outros trabalhos $20,22-24$, pois sabe-se que, na mulher, o 
controle de peso tem forte motivação estética e há maior preocupação com a saúde ${ }^{20}$. Ao comparar o estado nutricional de homens e mulheres, mostrouse que o peso pré-operatório, o maior peso antes da cirurgia e a CC foram significativamente maiores nos homens $(p<0,005)$, confirmando que as mulheres se preocupam mais com a saúde e procuram mais o serviço de saúde do que os homens.

Os dados da Pesquisa de Vigilância de Fatores de Risco e Proteção para Doenças Crônicas por Inquérito Telefônico ${ }^{25}$ também apontaram para um crescimento maior da obesidade entre os adultos de 35 e 44 anos, confirmando o encontrado no presente estudo e diferente da média de idade encontrada em um trabalho realizado também em Recife, em que prevalecia a população com idades entre 41 e 50 anos $(45,2 \%)^{23}$.

Em relação aos anos de estudo, predominaram os pacientes com 12 anos ou mais, dados semelhantes aos obtidos por Zyger et al. ${ }^{24}$ e que permite inferir que pessoas de maior escolaridade procuram mais o serviço de saúde, em comparação com pessoas de menor escolaridade ${ }^{26}$. Achados opostos foram encontrados por Rosa et al. ${ }^{27}$ e Rolim et al. ${ }^{23}$.

Em relação à prática de atividade física, 40\% dos pacientes eram ativos, como também observado por Boscatto et al. ${ }^{28}$. Essa predominância de indivíduos ativos pode ter ocorrido devido ao programa de acompanhamento do nosso Serviço realizado com esses pacientes previamente à cirurgia bariátrica. No entanto, observa-se ainda valores elevados de sedentários (31,7\%). O sedentarismo é um dos aspectos de estilo de vida que mais acometem a qualidade de vida dos obesos, sendo considerado fator de risco primário e independente para o desenvolvimento da obesidade. Ademais, também está relacionado com a qualidade dos alimentos ingeridos ${ }^{24}$. O sedentarismo, associado com o elevado consumo de açúcares simples e alimentos gordurosos, apresenta efeito de risco para a obesidade e para outras doenças crônicas. Tal associação foi observada no estudo ao relacionar a atividade física com o consumo alimentar, em que indivíduos sedentários apresentam o maior consumo de carboidratos $(p=0,041)$. Os carboidratos, por se tratarem de um grupo alimentar de aceitação e digestão mais fáceis, são frequentemente observados na dieta de indivíduos obesos 29,30 .

Dessa forma, o nível de atividade física possui relação significativa com o consumo de carboidratos, o que permite refletir sobre a importância da avaliação do consumo alimentar de indivíduos obesos candidatos à cirurgia, com o propósito de identificar e corrigir os erros alimentares. Outros estudos são necessários para verificar a possível associação do consumo alimentar com a composição corporal desses indivíduos.

\section{A B S T R A C T}

Objective: to evaluate the association of food consumption with nutritional status, physical activity and sociodemographic factors in the bariatric surgery period preoperative. Methods: we conducted a cross-sectional study from 2018 to 2019 , with patients admitted for bariatric surgery at the Oswaldo Cruz University Hospital, Federal University of Pernambuco. We collected data sociodemographic and nutritional data such as body mass index (BMI), waist circumference, highest weight and pre-surgical weight. We evaluated food consumption with the food frequency questionnaire based on the food pyramid adapted for bariatric patients and physical activity. Results: the study involved 60 patients, $78.3 \%$ were female, mean age was 38.8 \pm 9.6 years, 53.3\% were married, and 70\% had more than 12 years of education. As for physical activity, $31.7 \%$ were sedentary. Regarding nutritional status, the mean BMI was $47.3 \pm 6.96 \mathrm{~kg} / \mathrm{m}^{2}$. Preoperative weight, highest weight reached before surgery and waist circumference were higher in males $(p<0.05)$. There was an association between physical activity and food intake, and sedentary individuals consumed more carbohydrates $(p=0.041)$. On the other hand, sociodemographic data and nutritional status were not associated with food consumption. Conclusion: the level of physical activity showed significant association only with carbohydrate consumption.

Keywords: Bariatric Surgery. Obesity. Food Consumption. Nutritional Status. Preoperative Period. 


\section{REFERÊNCIAS}

1. Upadhyay J, Farr O, Perakakis N, Ghaly W, Mantzoros C. Obesity as a disease. Med Clin N Am. 2018;102(1):13-33.

2. Mechanick JI, Kushner RF, Sugerman HJ, GonzalezCampoy JM, Collazo-Clavell ML, Guven S, et al. American Association of Clinical Endocrinologist, The Obesity Society, and American Society for Metabolic \& Bariatric Surgery Medical Guidelines for Clinical Practice for the perioperative nutritional, metabolic and nonsurgical support of the bariatric surgery patient. Surg Obes Relat Dis. 2008;4(5 Suppl):S109-84. Erratum in: Surg Obes Relat Dis. 2010;6(1):112.

3. Acquafresca PA, Palermo M, Duza GE, Blanco LA, Serra EE. [Gastric Bypass versus Sleeve gastrectomy: comparison between type 2 Diabetes weight loss and complications. Review of randomized control trails]. Acta Gastroenterol Latinoam. 2015;45(2):143-54. Spanish.

4. Puzziferri N, Roshek TB 3rd, Mayo HG, Gallagher $\mathrm{R}$, Belle SH, Livingston $\mathrm{EH}$. Long-term follow-up after bariatric surgery: a systematic review. JAMA. 2014;312(9):934-42.

5. Sjöström L. Bariatric surgery and reduction in morbidity and mortality: experiences from the SOS study. Inter J Obes (Lond). 2008;32 Suppl 7: S93-7.

6. Maïmoun $L$, Lefebvre $P$, Aouinti $S$, Picot $M C$, Mariano-Goulart D, Nocca D; Montpellier Study Group of Bariatric Surgery. Acute and longer-term body composition changes after bariatric surgery. Surg Obes Relat Dis. Epub 2019 Jul 29.

7. Lupoli R, Lembo E, Saldalamacchia G, Avola CK, Angrisani L, Capaldo B. Bariatric surgery and long-term nutritional issues. World J Diabetes. 2017;8(11):464-74.

8. Bordalo LA, Teixeira TFS, Bressan J, Mourão DM. Cirurgia bariátrica: como e por que suplementar. Rev Assoc Med Bras. 2011;57(1):113-20.

9. Hall KD. The Potential Role of Protein Leverage in the US Obesity Epidemic. Obesity (Silver Spring). 2019;27(8):1222-4.
10. Ahmad D, Esmadi S, Hammad MH. Malnutrition secondary to non-compliance with vitamin and mineral supplements after gastric bypass surgery: what can we do about it? Am J Case Rep. 2012;13:209-13.

11. ABEP Associação Brasileira de Pesquisas. Critério de classificação econômica Brasil [Internet]. 2012 [Acesso 2019 mar 5]. Disponível em http://www. abep.org/criterio-brasil

12. Jellife DB. Evolución del estado de nutrición de la comunidad. 1 ed. Ginebra, Suiza: Organización Mundial de la Salud; 1968.

13. (WHO). Obesity: preventing and managing the global epidemic. Report of WHO Consultation on obesity. World Health Organization: Geneva; 1998.

14. Craig $C L$, Marshall Al, Sjöström $M$, Bauman $A E$, Booth $\mathrm{ML}$, Ainsworth $\mathrm{BE}$, et al. International physical activity questionnaire: 12-country reliability and validity. Med Sci Sports Exerc. 2003;35(8):1381-95.

15. Soares FL, Bissoni de Sousa L, Corradi-Perini C, Ramos da Cruz MR, Nunes MG, Branco-Filho AJ. Food Quality in the late postoperative period of bariatric surgery: an evaluation using the bariatric food pyramid. Obes Surg. 2014;24(9):1481-6.

16. Moizé VL, Pi-Sunyer $X$, Mochari $H$, Vidal J. Nutritional pyramid for post- gastric by-pass patients. Obes Surg. 2010;20(8):1133-41.

17. Fornés NS, Martins IS, Velásquez Melendez G, Latorre MRDO. Escores de consumo alimentar e níveis lipêmicos em população de São Paulo, Brasil. Rev. Saúde Pública. 2002;36(1):12-8.

18. Sociedade Brasileira de Cirurgia Bariátrica e Metabólica (SBCBM). Consenso Bariátrico. [Internet]. 2006 [Acesso em 2019 Aug 15]. Disponível em: http://www.sbcb.org.br/ membros_ consenso_bariatrico.php

19. Ribeiro de Moraes M, Lúcia de Mendonça Soares B, Maio R, Pessoa de Araújo Burgos MG. Clinicalnutritional evolution of older women submitted to Roux-en-Y gastric by-pass. Nutr Hosp. 2015;31(3):1330-5. 
20. Santos TD, Burgos MGPA, Lemos MCC, Cabral PC Aspectos clínicos e nutricionais em mulheres obesas durante o primeiro ano após bypass gástrico em Y-deRoux. ABCD Arq Bras Cir Dig. 2015;28(Suppl 1):56-60.

21. Trindade EM, Gebara TSS, Cambi MPC, Baretta GAP. Aspectos nutricionais e o uso de suplementos alimentares em mulheres submetidas ao bypass gástrico. ABCD Arq Bras Cir Dig. 2017;30(1):11-3.

22. Tedesco AK, Biazotto R, Gebara TSS, Cambi MPC, Baretta GAP. Pré e pós-operatório de cirurgia bariátrica: algumas alterações bioquímicas. $A B C D$ Arq Bras Cir Dig. 2016;29(Supl. 1):67-71.

23. Rolim FFA, Santa-Cruz F, Campos JM, Ferraz AAB. Repercussões em longo prazo da derivação gástrica em Y de Roux em população de baixa renda: avaliação após dez anos de cirurgia. Rev Col Bras Cir. 2018; 45(4):e1916.

24. Zyger LT, Zanardo VPS, Tomicki C. Perfil nutricional e estilo de vida de pacientes pré e pós-cirurgia bariátrica. Sci Med (Porto Alegre). 2016;26(3):ID23707.

25. Brasil. Ministério da Saúde. Secretaria de Vigilância em Saúde. Departamento de Analise em Saúde e Vigilância de Doenças não Transmissíveis. Vigitel Brasil 2018 - Vigilância de fatores de risco e proteção para doenças crônicas por inquérito telefônico: estimativas sobre frequência e distribuição sociodemográfica de fatores de risco e proteção para doenças crônicas nas capitais dos 26 estados brasileiros e no Distrito Federal em 2018. Brasília (DF): Ministério da Saúde; 2019.

26. Viacava F, Oliveira RAD, Carvalho CC, Laguardia J, Bellido JG. SUS: oferta, acesso e utilização de serviços de saúde nos últimos 30 anos. Ciên Saúde Coletiva. 2018;23(6):1751-62.
27. Rosa SC, Macedo JLS, Casulari LA, Canedo LR, Marques JVA. Perfil antropométrico e clínico de pacientes pós-bariátricos submetidos a procedimentos em cirurgia plástica. Rev Col Bras Cir. 2018;45(2):e1613.

28. Boscatto EC, Duarte MFS, Gomes MA. Estágios de mudança de comportamento e barreiras para atividade física em obesos mórbidos. Rev Bras Cineantropom Desempenho Hum. 2011;13(5):329-34.

29. Zaparolli M, Cruz MRR. Silova G, Radominsi R, Reichmann MTF, Schieferdecker ME, et al. Ingestão alimentar após cirurgia bariátrica: uma análise dos macronutrientes e adequação dos grupos alimentares à pirâmide específica. Nutr Clin Diet Hosp. 2018;38(1):36-9.

30. Castanho GKF, Marsola FC, Mclellan KCP, Nicola M, Moreto F, Burini RC. Consumo de frutas, verduras e legumes associado à Síndrome Metabólica e seus componentes em amostra populacional adulta. Ciênc Saúde Coletiva. 2013;18(2):385-92.

Recebido em: 09/10/2019

Aceito para publicação em: 29/10/2019

Conflito de interesse: nenhum.

Fonte de financiamento: nenhuma.

\section{Endereço para correspondência:}

Renata Adrielle Lima Vieira

E-mail: renata_adrielle@yahoo.com.br gburgos@hotlink.com.br

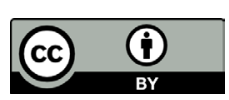

